\title{
p38MAPK inhibitor SB203580 sensitizes human SNU-C4 colon cancer cells to exisulind-induced apoptosis
}

\author{
SOO-JEONG LIM ${ }^{1,2^{*}}$, YOUNG-JU LEE ${ }^{2 *}$ and EUNMYONG LEE ${ }^{1}$ \\ ${ }^{1}$ Department of Bioscience and Biotechnology, Sejong University, Seoul; \\ ${ }^{2}$ Research Institute, National Cancer Center, Goyang, Gyeonggi, Korea
}

Received April 4, 2006; Accepted July 12, 2006

\begin{abstract}
Sulindac sulfone (exisulind), is a promising anticancer agent because of its ability to induce apoptosis in a variety of malignant cell types and its minimal toxicity to normal cells. The induction of apoptosis is thought to account for the growth inhibitory effect of exisulind. The mitogen-activated protein kinase (MAPK) cascade has been implicated in the regulation of apoptosis in response to exisulind. With human SNU-C4 colon cancer cells that were much more resistant to exisulind than other colon cancer cells, in this study, we investigated whether the modulation of MAPK activity by using selective MAPK inhibitors can contribute to sensitizing SNU-C4 cells to exisulind. Exisulind (400 and $600 \mu \mathrm{M}$ ) slightly increased the phosphorylation of pERK1/2 but pretreatment with the pERK1/2 inhibitor PD98059 did not significantly change the apoptotic response of SNU-C4 cells. The same doses of exisulind increased the phosphorylation of p38MAPK, and pretreatment with the p38MAPK inhibitor SB203580 significantly potentiated growth inhibition and apoptosis induced by exisulind in SNU-C4 cells. We further found that apoptosis induced by a combination of exisulind and SB203580 was mediated through caspase activation. Collectively, our findings indicate that selective p38MAPK inhibitors potentiate apoptosis induction by exisulind in SNU$\mathrm{C} 4$ cells. Such combinations may provide a more effective and less toxic strategy for the prevention or treatment of colon cancer.
\end{abstract}

\section{Introduction}

The nonsteroidal anti-inflammatory drug sulindac inhibits chemical carcinogenesis in rodents and causes the regression

Correspondence to: Dr Soo-Jeong Lim, Department of Bioscience and Biotechnology, Sejong University, 98 Kunja-dong, Kwangjin-gu, Seoul 143-747, Korea

E-mail: sjlim@sejong.ac.kr

${ }^{*}$ Contributed equally

Key words: exisulind, p38MAPK, apoptosis, colon cancer, SB203580 of adenomas in patients with familial adenomatous polyposis coli of the colon. Sulindac is intracelluarly metabolized to a sulfide derivative with cyclooxygenase (COX) inhibitory activity as well as to a sulfone derivative without COX inhibitory activity. However, sulindac sulfone (exisulind) also induces apoptosis in a wide range of human tumor cell lines, but does not affect normal cells (1). Furthermore, it inhibits the growth of human prostate cancer cells in nude mice (2). In clinical trials, exisulind prevented colorectal polyp formation in patients with familial adenomatous polyposis. The induction of apoptosis is thought to be a major biological mechanism of growth inhibition by sulindac metabolites in cultured cells, animal models, and adenomas in patients with familial adenomatous polyposis.

The role of mitogen-activated protein kinase (MAPK) cascade has been implicated in signal transduction and the regulation of cell survival and death response to diverse external stimuli $(3,4)$. The MAPK family is composed of three types of serine/threonine kinases: Extracellular signalregulated kinases (ERKs), c-Jun $\mathrm{NH}_{2}$-terminal kinases (JNKs), and p38MAPK. These MAPKs mediate cellular responses to environmental stresses such as proinflammatory cytokines, UV light, $\gamma$-irradiation, growth factor withdrawal, ceramide, protein synthesis inhibitors, heat shock, osmotic shock, and anticancer drugs $(3,5,6)$, and they have been implicated in the process of cell death in many cell types $(7,8)$. Exisulind-induced apoptosis has been found to involve the inhibition of ERK1/2 in human colon cancer cells (9-11) In other studies, exisulind-induced apoptosis was shown to be mediated by JNK activation, which seems likely to be a downstream event of cellular cyclic GMP accumulation and the subsequent activation of the cyclic GMP-dependent protein kinase (12-14). The role of p38MAPK in regulating exisulind-induced apoptosis currently remains unclear but a recent study showing that a selective p38MAPK inhibitor potentiated apoptosis induction by sulindac sulfide suggests a plausible role of p38MAPK in mediating cellular response against sulindac analogues (15). We sought to investigate whether the modulation of MAPK activity by pharamacological MAPK inhibitors can sensitize exisulind-resistant SNU-C4 colon cancer cells to exisulind treatment. We provide experimental evidence that selective inhibitors of p38MAPK potentiated apoptosis induction by exisulind in human SNU-C4 colon cancer cells. 


\section{Materials and methods}

Cell lines and cultures. The human HCT116 and LoVo colon cancer cell lines were purchased from the American Type Culture Collection (Manassas, VA, USA), and the human SNU-C4 colon cancer cell line was purchased from the Korean Cell Line Bank (Seoul, Korea). Cells were cultured in RPMI-1640 medium supplemented with $10 \%$ heatinactivated fetal bovine serum (Hyclone, Logan, UT, USA) and $100 \mathrm{U} / \mathrm{ml}$ of both penicillin and streptomycin. Cells were grown in incubators at $37^{\circ} \mathrm{C}$ in a humid atmosphere of $95 \%$ air $/ 5 \% \mathrm{CO}_{2}$.

Reagents and antibodies. Antibodies to human B-actin and 3(4,5-dimethylthiazol-2-yl)-2,5-diphenyl-tetrazolium bromide (MTT) were obtained from Sigma (St. Louis, MO, USA). Exisulind was obtained from LKT Laboratories (St. Paul, MN, USA). SB203580 and PD98059 were purchased from Calbiochem (La Jolla, CA, USA). $N$-benzyloxycarbonyl-ValAla-Asp-fluoromethylketone (z-VAD-FMK) was obtained from Enzyme System Products (Livemore, CA, USA). Antibodies to human poly(ADP-ribose) polymerase (PARP) were purchased from BD Pharmingen (San Diego, CA, USA), and the antibody to active caspase- 3 was obtained from Cell Signaling Technology (Beverly, MA, USA). Phosphospecific and nonphosphospecific p38MAPK antibodies were obtained from New England Biolabs (Beverly, MA, USA). Phosphospecific and nonphosphospecific pERK1/2 antibodies were obtained from Cell signaling Technology. All other chemicals were of reagent grade and used without further purification.

Analysis of cell viability. Cells were seeded into 96-well plates. The following day, various doses of exisulind (diluted from a $500 \mathrm{mM}$ stock in DMSO) or vehicle alone were added. The cells were then incubated for $48 \mathrm{~h}$, and cell growth and viability were measured using MTT. The ability of cells to form formazan crystals by active mitochondrial respiration was determined using a microplate spectrophotometer (Molecular Devices) after dissolving the crystals in DMSO.

Assay of apoptosis. Following experimental treatments, the cell growth medium was harvested. After trypsinization, apoptotic bodies were collected by centrifugation, pooled with cells, and resuspended in Dulbecco's PBS. Histoneassociated DNA fragments were quantified using a photometric enzyme immunoassay using the Cell Death Detection ELISA $^{\text {plus }}$ kit (Roche Applied Bioscience according to the manufacturer's protocol) (16).

Immunoblotting. Treated cells were collected by scraping, washed twice with PBS, and incubated for 15 to $30 \mathrm{~min}$ on ice in a lysis buffer containing $150 \mathrm{mM} \mathrm{NaCl}, 10 \mathrm{mM}$ Tris, $0.2 \%$ Triton X-100, 0.3\% NP-40, $0.2 \mathrm{mM} \mathrm{Na}_{3} \mathrm{VO}_{4}$, and protease inhibitors (Roche Diagnostics) (pH 7.4). Aliquots of cell lysates containing equal amounts of protein were denatured in SDS-reducing buffer by boiling for $5 \mathrm{~min}$, resolved on SDS-polyacrylamide gels, and transferred to nitrocellulose membranes. The membranes were blocked with $5 \%$ nonfat dry milk and then probed with specific primary antibodies, followed by incubation with appropriate
(A)

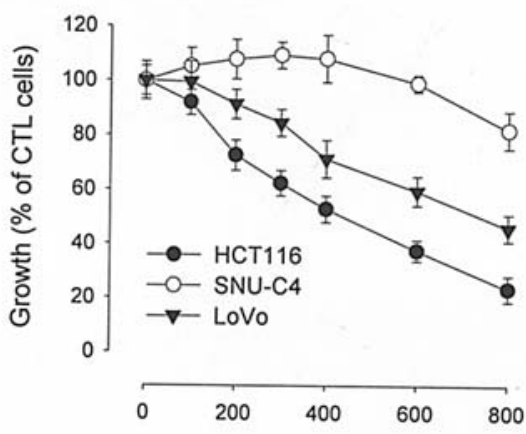

(B)
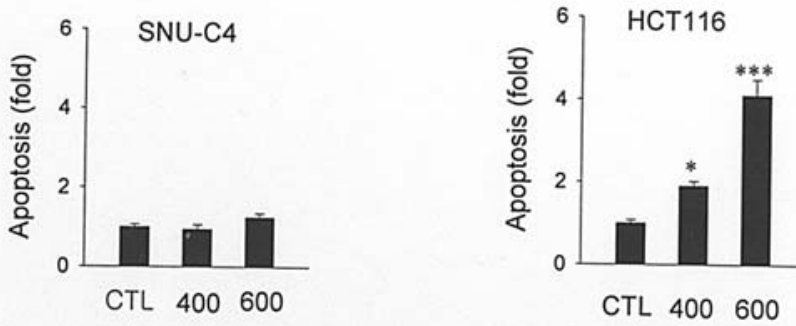

Figure 1. Exisulind differentially induced growth inhibition and apoptosis in human HCT116 and SNU-C4 colon cancer cell lines. (A) Cells were seeded in 96-well plates (4-6x10 cells/well) and, starting $24 \mathrm{~h}$ later, were treated with varying doses of exisulind or DMSO vehicle. At 48-h postincubation, the growth and viability of the cells were determined by MTT assay. Results are expressed as the percentage growth relative to control (DMSO) cells (mean \pm SD of two experiments performed in triplicate). (B) Apoptosis was quantified by an ELISA that specifically detected histone-associated DNA. Exponentially growing cells were treated with 400 and $600 \mu \mathrm{M}$ doses of exisulind, and subjected to apoptosis analysis. The bar represents the ratio of the absorbance at $405 \mathrm{~nm}$ in cells incubated with exisulind and in control cells (mean $\pm \mathrm{SD}$ of two experiments performed in triplicate). $\left({ }^{*} \mathrm{p}<0.05\right.$; ${ }^{* * *} \mathrm{p}<0.001$ by unpaired t-test, compared with control cells)

peroxidase-conjugated secondary antibodies. The blots were developed with ECL Plus reagent (Amersham, Arlington Heights, IL, USA) according to the manufacturer's protocol.

Statistical analysis. The significance of differences between the values obtained under different experimental conditions was determined using a two-tailed unpaired Student's t-test. Values of $\mathrm{p}<0.05$ were considered statistically significant.

\section{Results}

Exisulind resistance in SNU-C4 cells. We first treated HCT116, LoVo and SNU-C4 colon cancer cells with increasing doses of exisulind for $48 \mathrm{~h}$. We found that $100-800 \mu \mathrm{M}$ exisulind dose-dependently inhibited the growth of HCT116 and LoVo cells, but not SNU-C4 cells (Fig. 1A). Therefore, the dose of exisulind resulting in 50\% inhibition of growth was $\sim 450$ and $700 \mu \mathrm{M}$ in HCT116 and LoVo cells, but even higher than $1000 \mu \mathrm{M}$ in SNU-C4 cells.

Because growth inhibition by exisulind is known to be associated with the induction of apoptosis, we compared the 
(A)

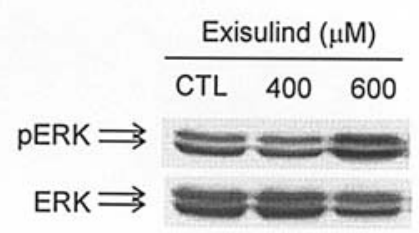

(B)

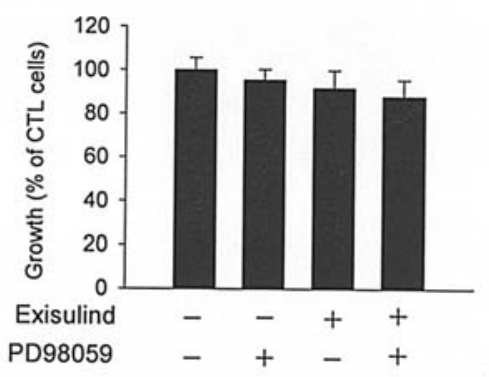

(C)

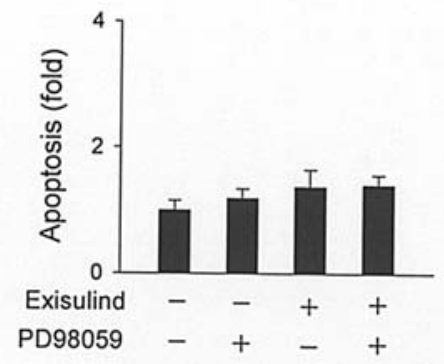

Figure 2. Effect of the pERK1/2 inhibitor PD98059 on the exisulind-induced apoptosis in SNU-C4 cells. (A) Changes in the phosphorylated pERK1/2 by exisulind treatment. SNU-C4 cells were treated with 400 and $600 \mu \mathrm{M}$ exisulind. At 24-h postincubation, cellular lysates were obtained and subjected to immunoblot analysis to detect phosphoactive forms of pERK1/2. (B) With or without 1-h pretreatment with $25 \mu \mathrm{M}$ PD98059, SNU-C4 cells were treated with $600 \mu \mathrm{M}$ exisulind for $48 \mathrm{~h}$, and then subjected to MTT assay. Results are expressed as the percentage growth relative to control (DMSO) cells. (C) Apoptosis was quantified by an ELISA that specifically detected histone-associated DNA. With or without 1-h pretreatment with $25 \mu \mathrm{M}$ PD98059, cells were treated with $600 \mu \mathrm{M}$ exisulind for $48 \mathrm{~h}$. The bar represents the ratio of the absorbance at $405 \mathrm{~nm}$ in cells incubated with exisulind, PD98059 or exisulind plus PD98059, and that in control (DMSO) cells (mean \pm SD of two experiments performed in triplicate).

extent of apoptosis in exisulind-resistant SNU-C4 cells with the most sensitive HCT116 cells. Analysis of DNA fragmentation revealed that exisulind doses that induce growth inhibition in HCT116 cells but not in SNU-C4 cells (400 and $600 \mu \mathrm{M}$ ) caused a dose-dependent induction of apoptosis in HCT116 cells but not in SNU-C4 cells (Fig. 1B). In addition, exisulind caused morphological changes, including cell shrinkage and chromatin condensation, in HCT116 cells but not in SNU-C4 cells (data not shown). These results indicate that SNU-C4 cells are resistant to exisulind-induced apoptosis.

Effect of pERK1/2 inhibitors on exisulind response in SNU-C4 cells. To investigate the role of pERK $1 / 2$ in exisulindinduced apoptosis, we monitored the dose-dependent changes in pERK $1 / 2$ phosphorylation, which reflects its enzyme activity. When SNU-C4 cells were treated with 400 or $600 \mu \mathrm{M}$ exisulind for $24 \mathrm{~h}$, the phosphorylation of pERK1/2 was slightly increased by $600 \mu \mathrm{M}$ exisulind (Fig. 2A). Pretreatment with a pERK1/2 inhibitor PD98059 did not affect the growth inhibition (Fig. 2B) and apoptosis (Fig. 2C) induced by $600 \mu \mathrm{M}$ exisulind. Our data suggest that pERK1/2 inhibition is not sufficient to sensitize SNU-C4 cells to exisulind-induced apoptosis.

Effect of p38MAPK inhibitors on exisulind response in SNU-C4 cells. To investigate the role of p38MAPK in exisulindinduced apoptosis, we monitored the dose-dependent change in p38MAPK phosphorylation. Exisulind (400 and $600 \mu \mathrm{M}$ ) caused a dose-dependent increase in the phosphorylation of p38MAPK, suggesting the activation of this protein kinase. In contrast, in exisulind-sensitive HCT116 cells, $600 \mu \mathrm{M}$ exisulind caused a significant decrease in the level of phosphorylated p38MAPK without changes in its expression (Fig. 3A). To further investigate the role of p38MAPK in exisulind-induced apoptosis, we pretreated cells with the p38MAPK inhibitor SB203580. This inhibitor significantly augmented exisulind-induced growth inhibition and apoptosis in SNU-C4 cells (Fig. 3B and C). These data suggest that p38MAPK inhibition can sensitize SNU-C4 cells to exisulind treatment.

Caspase dependency in mediating apoptosis induced by exisulind and SB203580. Exisulind-induced apoptosis has been shown to involve the activation of the caspase cascade $(10,17)$. We therefore examined whether apoptosis induced by exisulind in combination with SB2030580 involved caspase activation by measuring the conversion of procaspase3 to its catalytically active form (p17/p19). The catalytically active form of caspase-3 was evident in exisulind-treated SNU-C4 cells only when they were treated with the exisulind and SB203580 combination (Fig. 4A). We also detected the 85-kDa PARP fragment, a general marker of caspasedependent apoptosis, only when SNU-C4 cells were treated with both exisulind and SB203580 for 48 h (Fig. 4A). Finally, we found that pretreatment with a general caspase inhibitor z-VAD-FMK significantly blocked the apoptosis induced by the exisulind and SB203580 combination (Fig. 4B). Therefore, it seems likely that apoptosis induced by combination treatment with exisulind and SB203580 is mediated through caspase activation.

\section{Discussion}

Exisulind reduces cell viability and induces apoptosis that may be critical to its anticancer effects. Earlier studies have shown that the modulation of MAPK pathways is involved in the regulation of the apoptotic response of cells to exisulind, and that the inhibition of ERK1/2 activity by pharmacological inhibitors augmented the exisulind-induced apoptosis in human HCT116 colon cancer cells (9). Our present data suggest that the pERK1/2 inhibitor PD98059 was ineffective in increasing the apoptotic susceptibility of SNU-C4 cells, suggesting that the role of the MAPK pathway in exisulindinduced apoptosis may differ depending on the cellular context as well as cell types. To date, the role of p38MAPK in exisulind-induced apoptosis remains unknown. Here we 
(A)

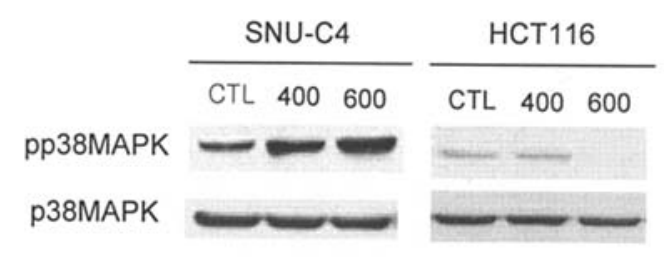

(B)

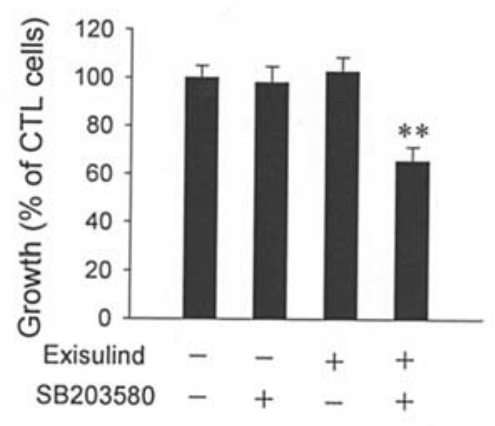

(C)

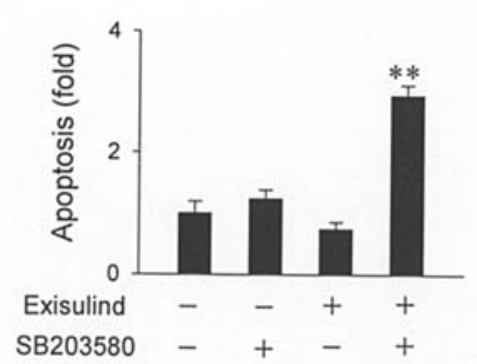

Figure 3. Effect of the p38MAPK inhibitor PD98059 on the exisulindinduced apoptosis. (A) Changes in the phosphorylated p38MAPK by exisulind treatment. HCT116 and SNU-C4 cells were treated with indicated concentrations of exisulind. At 24-h postincubation, cellular lysates were obtained and subjected to immunoblot analysis to detect phosphoactive forms of p38MAPK. (B) With or without 1-h pretreatment with $20 \mu \mathrm{M}$ SB203580, SNU-C4 cells were treated with $600 \mu \mathrm{M}$ exisulind for $48 \mathrm{~h}$, and then subjected to MTT assay. Results are expressed as the percentage growth relative to control (DMSO) cells. (C) Apoptosis was quantified by an ELISA that specifically detected histone-associated DNA. With or without 1-h pretreatment with $20 \mu \mathrm{M} \mathrm{SB} 203580$, cells were treated with $600 \mu \mathrm{M}$ exisulind for $48 \mathrm{~h}$. The bar represents the ratio of the absorbance at $405 \mathrm{~nm}$ in cells incubated with exisulind, SB203580 or exisulind plus SB203580, and that in control (DMSO) cells (mean \pm SD of two experiments performed in triplicate) ${ }^{* *} \mathrm{p}<0.01$ by unpaired $\mathrm{t}$-test, compared with control (DMSO) cells]

observed the dose-dependent increase in the phosphorylation of p38MAPK by exisulind treatment in exisulind-resistant SNU-C4 cells in contrast to the decrease in exisulindsensitive HCT116 cells. Furthermore, our present data indicate that the p38MAPK inhibitor SB203580 greatly augmented the apoptotic response of SNU-C4 cells to exisulind treatment while SB203580 alone had no significant effect on growth or apoptosis. In accordance with our data, a recent study showed that $\mathrm{p} 38 \mathrm{MAPK}$ inhibition increased the apoptotic susceptibility of HCA-7 colon cancer cells to sulindac sulfide, another sulindac metabolite whose antitumor effects are associated with its apoptosis-inducing ability (15). Therefore, p38MAPK activation may inhibit the apoptosis induced by sulindac metabolites.
(A)

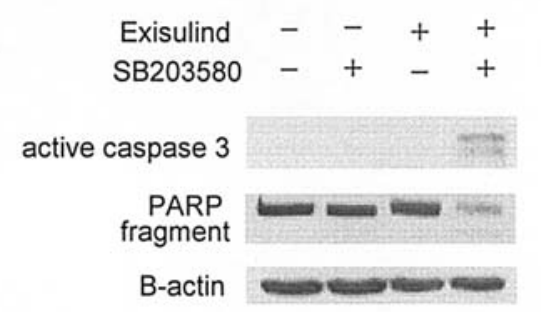

(B)

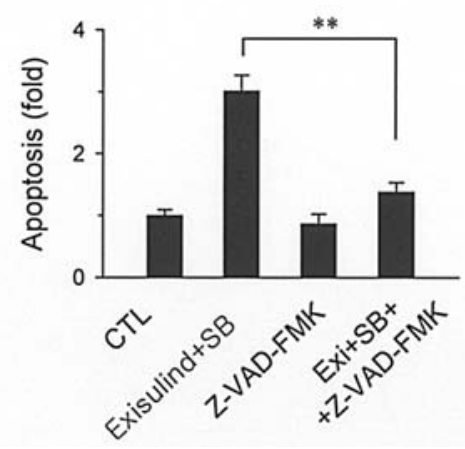

Figure 4. Combination of p38MAPK inhibitor PD98059 and exisulind induced caspase-dependent apoptosis in SNU-C4 cells. (A) Imunoblot analysis for detecting PARP cleavage and caspase activation. With or without 1-h pretreatment with $20 \mu \mathrm{M} \mathrm{SB} 203580$, cells were treated with $600 \mu \mathrm{M}$ exisulind for $48 \mathrm{~h}$. Cell lysates were subjected to immunoblotting with appropriate antibodies. Immunoblotting with an antibody to $\beta$-actin was used to control for equal loading of proteins per lane. (B) Effect of pretreatment with a general caspase inhibitor $\mathrm{z}-\mathrm{VAD}$-fmk on the apoptotic cell death induced by combination treatment with exisulind and PD98059. After 2-h pretreatment with $25 \mu \mathrm{M}$ of the pan-caspase inhibitor, z-VAD-fmk, SNU-C4 cells were treated with exisulind $(600 \mu \mathrm{M})$ and SB203580 $(20 \mu \mathrm{M})$ for $48 \mathrm{~h}$. Apoptosis was quantified by an ELISA that specifically detected histone-associated DNA. The bar represents the ratio of the absorbance at $405 \mathrm{~nm}$ in cells treated as indicated and in control (DMSO) cells $\left({ }^{* *} \mathrm{p}<0.01\right.$ by unpaired t-test).

The mechanism by which p38MAPK modulates apoptosis is poorly understood. Depending on the apoptotic stimuli and the cellular context, p38MAPK has been suggested to have an antiapoptotic and protective role. p38MAPK activation contributed to the execution of apoptosis in response to certain types of agents, such as the tumor necrosis factoralpha (18), zinc (19), and sodium arsenite (20). On the contrary, the inhibition of p38MAPK was involved in staurosporine-induced apoptosis (21), and p38MAPK inhibitors promoted TNF-alpha-induced apoptosis in human myelomonocytic leukemia cells (22). Our results do not establish how the inhibition of p38MAPK leads to caspase activation, PARP cleavage, and execution of the apoptotic process in exisulind-resistant SNU-C4 cells. A recent study, however, showed that the inhibition of p38MAPK can inhibit the expression of Bcl-2 and sensitize cells to drug-induced apoptosis, suggesting that p38MAPK plays a key role in drug resistance $(23,24)$. Therefore, we speculate that p38MAPK regulates the expression of antiapoptotic proteins such as Bcl-2 in exisulind-treated colon cancer cells and, that the 
pharmacological inhibition of p38MAPK also leads to the decreased expression of antiapoptotic proteins in exisulindresistant SNU-C4 cells. Further studies are needed, however, to test the validity of this hypothesis.

Previous studies have shown that JNK activation is a major mediator of exisulind-induced apoptosis $(12,14)$. The inhibition of p38MAPK has been shown to be linked to JNK activation in staurosporine-induced apoptosis (21). Therefore, we tested whether the enhancement of exisulindinduced apoptosis by SB203580 is associated with an increased activation of JNK. However, we did not observe a significant increase in JNK activation under these conditions (data not shown), suggesting that the molecular pathway by which exisulind causes apoptosis may be different under conditions where p38MAPK is inhibited.

Recently, considerable attention has been focused on the use of combinations of anticancer agents for prevention or enhanced efficacy. Such combinations can lower the necessary doses of each agent, reducing their toxicity. Attempts have been made to test the interaction of exisulind with other anticancer agents. For example, exisulind has been shown to exert a synergistic anticancer effect when used in combination with the proteasome inhibitor bortezomib (25), the green tea component epigallocatechin gallate (26), and chemotherapeutic drugs including docetaxel (27) and cisplatin (28). Therefore, our results may provide a molecular basis for the rational design of combination strategies using exisulind and agents that reduce p38MAPK activity for the prevention and treatment of colon cancer.

\section{Acknowledgements}

This study was supported by a research grant from the National Cancer Center 0310010-3.

\section{References}

1. Stoner GD, Budd GT, Ganapathi R, et al: Sulindac sulfone induced regression of rectal polyps in patients with familial adenomatous polyposis. Adv Exp Med Biol 470: 45-53, 1999.

2. Goluboff ET, Shabsigh A, Saidi JA, et al: Exisulind (sulindac sulfone) suppresses growth of human prostate cancer in a nude mouse xenograft model by increasing apoptosis. Urology 53: 440-445, 1999.

3. Whitmarsh AJ and Davis RJ: Transcription factor AP-1 regulation by mitogen-activated protein kinase signal transduction pathways. J Mol Med 74: 589-607, 1996.

4. Milella M, Precupanu CM, Gregorj C, et al: Beyond single pathway inhibition: MEK inhibitors as a platform for the development of pharmacological combinations with synergistic anti-leukemic effects. Curr Pharm Des 11: 2779-2795, 2005.

5. English J, Pearson G, Wilsbacher J, et al: New insights into the control of MAP kinase pathways. Exp Cell Res 253: 255-270, 1999.

6. Lee JS, Lee JJ and Seo JS: HSP70 deficiency results in activation of c-Jun N-terminal Kinase, extracellular signalregulated kinase, and caspase- 3 in hyperosmolarity-induced apoptosis. J Biol Chem 280: 6634-6641, 2005.

7. Lieu CH, Liu CC, Yu TH, et al: Role of mitogen-activated protein kinase in taxol-induced apoptosis in human leukemic U937 cells. Cell Growth Differ 9: 767-776, 1998.

8. Xu C, Shen G, Yuan X, et al: ERK and JNK signaling pathways are involved in the regulation of activator protein 1 and cell death elicited by three isothiocyanates in human prostate cancer PC-3 cells. Carcinogenesis 273: 435-445, 2005.
9. Rice PL, Goldberg RJ, Ray EC, et al: Inhibition of extracellular signal-regulated kinase $1 / 2$ phosphorylation and induction of apoptosis by sulindac metabolites. Cancer Res 61: 1541-1547, 2001.

10. Rice PL, Kelloff J, Sullivan H, et al: Sulindac metabolites induce caspase- and proteasome-dependent degradation of betacatenin protein in human colon cancer cells. Mol Cancer Ther 2: 885-892, 2003.

11. Rice PL, Peters SL, Beard KS, et al: Sulindac independently modulates extracellular signal-regulated kinase $1 / 2$ and cyclic GMP-dependent protein kinase signaling pathways. Mol Cancer Ther 5: 746-754, 2006.

12. Soh JW, Mao Y, Kim MG, et al: Cyclic GMP mediates apoptosis induced by sulindac derivatives via activation of c-Jun NH2-terminal kinase 1. Clin Cancer Res 6: 4136-4141, 2000.

13. Thompson WJ, Piazza GA, Li H, et al: Exisulind induction of apoptosis involves guanosine $3^{\prime}, 5^{\prime}$-cyclic monophosphate phosphodiesterase inhibition, protein kinase $\mathrm{G}$ activation, and attenuated beta-catenin. Cancer Res 60: 3338-3342, 2000.

14. Czibere A, Prall WC, Zerbini LF, et al: The nonsteroidal antiinflammatory drug Exisulind selectively induces apoptosis via JNK in secondary acute myeloid leukemia after myelodysplastic syndrome. Cell Cycle 4: 812-817, 2005.

15. Sun Y and Sinicrope FA: Selective inhibitors of MEK1/ ERK44/42 and p38 mitogen-activated protein kinases potentiate apoptosis induction by sulindac sulfide in human colon carcinoma cells. Mol Cancer Ther 4: 51-59, 2005.

16. Yin Z, Patel SJ, Wang WL, et al: Peptide inhibitors of dengue virus NS3 protease. Part 1: Warhead. Bioorg Med Chem Lett 16: 40-43, 2005.

17. Li H, Liu L, David ML, et al: Pro-apoptotic actions of exisulind and CP461 in SW480 colon tumor cells involve beta-catenin and cyclin D1 down-regulation. Biochem Pharmacol 64: 1325-1336, 2002.

18. Valladares A, Alvarez AM, Ventura JJ, et al: p38 mitogenactivated protein kinase mediates tumor necrosis factor-alphainduced apoptosis in rat fetal brown adipocytes. Endocrinology 141: 4383-4395, 2000.

19. Kondoh M, Tasaki E, Araragi S, et al: Requirement of caspase and p38MAPK activation in zinc-induced apoptosis in human leukemia HL-60 cells. Eur J Biochem 269: 6204-6211, 2002.

20. Kim JY, Choi JA, Kim TH, et al: Involvement of p38 mitogenactivated protein kinase in the cell growth inhibition by sodium arsenite. J Cell Physiol 190: 29-37, 2002.

21. Zhang H, Vollmer M, De Geyter M, et al: Apoptosis and differentiation induced by staurosporine in granulosa tumor cells is coupled with activation of JNK and suppression of p38 MAPK. Int J Oncol 26: 1575-1580, 2005.

22. Nakada S, Kawano T, Saito-akita S, et al: MEK and p38MAPK inhibitors potentiate TNF-alpha induced apoptosis in U937 cells. Anticancer Res 21: 167-171, 2001.

23. Vega MI, Huerta-Yepaz S, Garban H, et al: Rituximab inhibits p38 MAPK activity in 2F7 B NHL and decreases IL-10 transcription: pivotal role of p38 MAPK in drug resistance. Oncogene 23: 3530-3540, 2004.

24. Lin JC, Chang SY, Hsieh DS, et al: Modulation of mitogenactivated protein kinase cascades by differentiation-1 protein: acquired drug resistance of hormone independent prostate cancer cells. J Urol 174: 2022-2026, 2005.

25. Minami T, Adachi M, Kawamura R, et al: Sulindac enhances the proteasome inhibitor bortezomib-mediated oxidative stress and anticancer activity. Clin Cancer Res 11: 5248-5256, 2005.

26. Ohishi T, Kishimoto Y, Miura N, et al: Synergistic effects of (-)-epigallocatechin gallate with sulindac against colon carcinogenesis of rats treated with azoxymethane. Cancer Lett 177: 49-56, 2002

27. Chan DC, Earle KA, Zhao TL, et al: Exisulind in combination with docetaxel inhibits growth and metastasis of human lung cancer and prolongs survival in athymic nude rats with orthotopic lung tumors. Clin Cancer Res 8: 904-912, 2002.

28. Soriano AF, Helfrich B, Chan DC, et al: Synergistic effects of new chemopreventive agents and conventional cytotoxic agents against human lung cancer cell lines. Cancer Res 59: 6178-6184, 1999. 